\title{
Highly accelerated 3D myocardial late fadolinium enhancement MRI using ESPIRiT compressed sensing: initial feasibility
}

\author{
Peng Lai ${ }^{*}$, Piero Ghedin², Gianluca Pontone ${ }^{3}$, Anja Brau² \\ From 17th Annual SCMR Scientific Sessions \\ New Orleans, LA, USA. 16-19 January 2014
}

\section{Background}

Myocardial late gadolinium enhancement (LGE) MRI has become a standard clinical exam for characterizing myocardial viability after infarction and for assessing many nonischemic myocardial diseaseses. Typically, a $3 \mathrm{D}$ slab covering the myocardium is scanned within a single breath-hold. However, 3D LGE with sufficient resolution and slice coverage requires a long scan time and is challenging in cardiac patients with limited breath-hold capability. Recently, compressed sensing (CS) [1] has demonstrated the ability to further accelerate MRI on top of parallel imaging (PI) and has demonstrated promising results for 3D LGE [2]. This work intended to evaluate the feasibility of a CS-PI method based on ESPIRiT [3-5] and optimize its data acquisition for improving the robustness of 3D LGE with high acceleration.

\section{Methods}

k-space sampling: As shown in Figure 1, a variable density Poisson disk sampling (VDPDS) scheme was used for CS acceleration, with variable acceleration depending on offcenter distance and Poisson disk distribution at each local $\mathrm{k}$-space region. For accurate reconstruction, the acceleration quadratically increases from center to outer k-space. Partial Fourier along ky was used to further reduce scan time. Image reconstruction: As described in ESPIRiT [3-5], first, coil sensitivity was estimated from calibration data-based eigenvector computations. Next, k-space was completed by iteratively enforcing coil sensitivity and

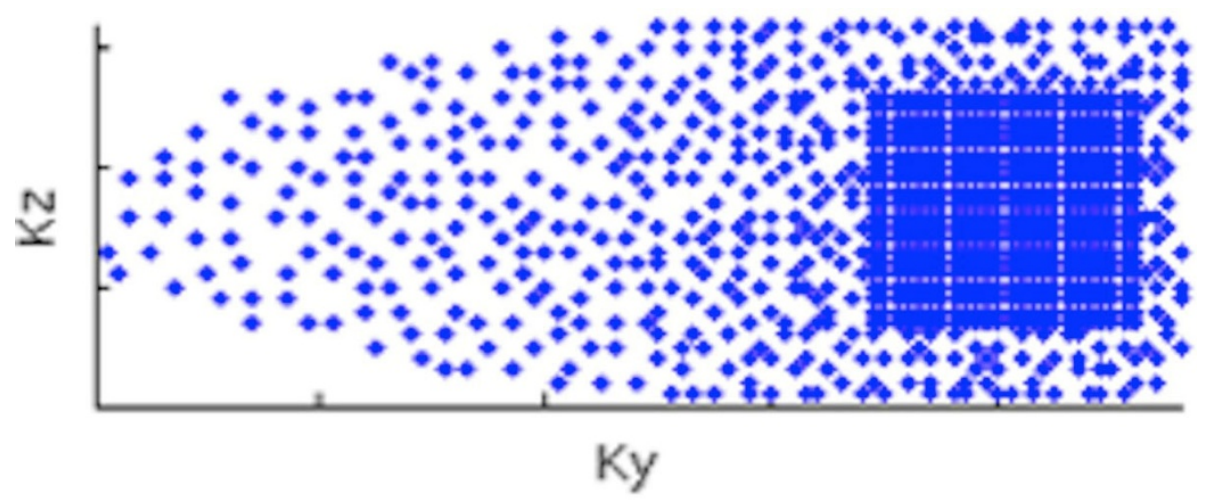

Figure 1 Variable density Poisson disk sampling for ESPIRiT.

'MR Applications and Workflow, GE Healthcare, Menlo Park, California, USA

Full list of author information is available at the end of the article

(c) 2014 Lai et al.; licensee BioMed Central Ltd. This is an Open Access article distributed under the terms of the Creative Commons 


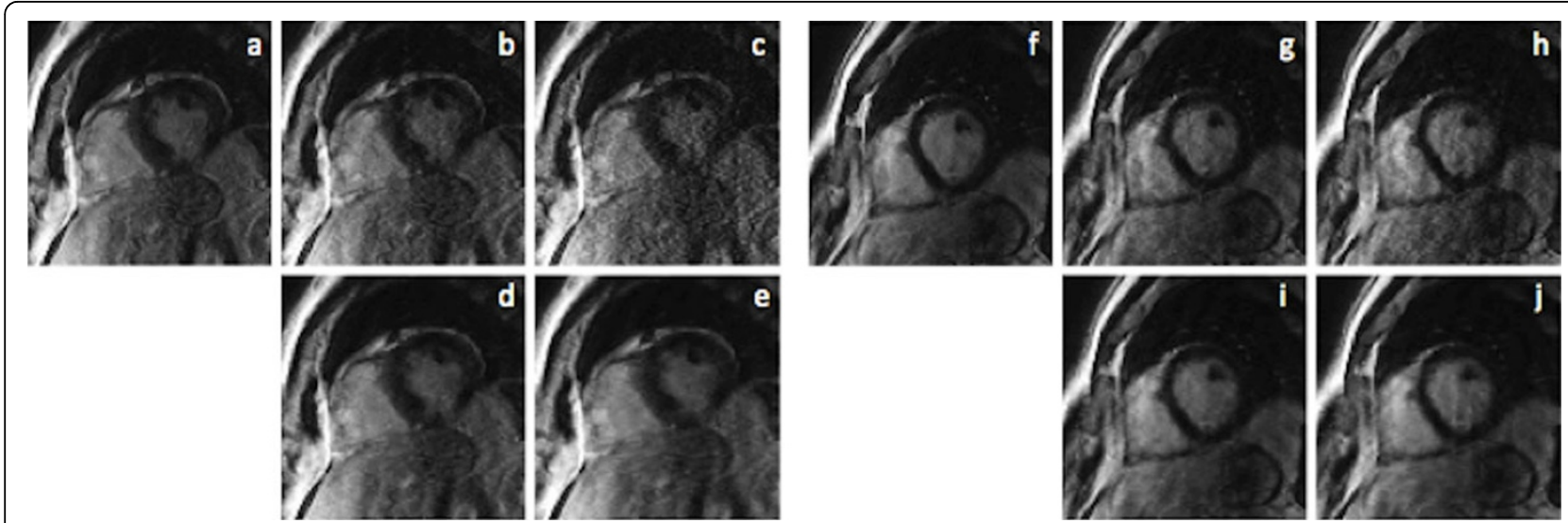

Figure 2 Comparison of different accelerations and reconstructions in case 1 (left) and case 2 (right). Full k-space image (a, $f$ ) and simulated reconstruction using ARC with $2 \times 2(b, g) \& 3 \times 2(c, h)$ and ESPIRiT with net acceleration same as ARC $2 \times 2(d, i) \& 3 \times 2(e, j)$.

signal sparsity until convergence. Full-kspace 3D LGE datasets were collected from 3 patients on a GE $1.5 \mathrm{~T}$ system using 3D FGRE and an 8 channel cardiac coil. Typical imaging parameters included: $15^{\circ}$ flip angle, matrix size of $200 \times 200,14$ slices with $10 \mathrm{~mm}$ thickness, TR/TE $=$ 2.5/1.1 ms, partial Fourier factor of 0.6 along ky, $28 \mathrm{~s}$ scan time. TI was determined on a per-patient basis from a TI scout scan. Full k-space was downsampled offline using the VDPDS scheme and reconstructed using ESPIRiT. For comparison, conventional autocalibrated PI was also simulated using regular $2 \mathrm{D}$ acceleration and reconstructed using a GE product reconstruction (ARC).

\section{Results}

Figure 2 shows 2 representative cases. In case 1 (left), ARC (b, c) produces substantial noise amplification and visible aliasing artifacts at these high accelerations, which interfere with pathology at inferoposterior endocardium. In comparison, ESPIRiT (d, e) generates image quality and delineation of the delayed enhancement comparable to full k-space image (a), though with slight edge blurring. In another case (right), similarly, ESPIRiT (i, j) significantly suppresses artifacts and provides superior SNR vs. ARC (g, h).

\section{Conclusions}

In this initial study, ESPIRiT with VDPDS was shown to enable higher acceleration with better image quality than conventional PI and is a promising approach for improving the robustness of 3D LGE by shortening breathholding time.

\section{Authors' details}

${ }^{1}$ MR Applications and Workflow, GE Healthcare, Menlo Park, California, USA. ${ }^{2}$ MR Applications and Workflow, GE Healthcare, Garching, Munchen, Germany. ${ }^{3}$ Department of Cardiovascular Imaging, Centro Cardiologico Monzino, Milan, Italy.
Published: 16 January 2014

\section{References}

1. Lustig: MRM 2007, 58.

2. Akcakaya: Rad 2012, 264.

3. Lai: ISMRM 2010, 345.

4. Lai: ISMRM 2011, 68.

5. Uecker: MRM, online.

doi:10.1186/1532-429X-16-S1-W25

Cite this article as: Lai et al: Highly accelerated 3D myocardial late fadolinium enhancement MRI using ESPIRiT compressed sensing: initial feasibility. Journal of Cardiovascular Magnetic Resonance 2014 16(Suppl 1): W25.

Submit your next manuscript to BioMed Central and take full advantage of:

- Convenient online submission

- Thorough peer review

- No space constraints or color figure charges

- Immediate publication on acceptance

- Inclusion in PubMed, CAS, Scopus and Google Scholar

- Research which is freely available for redistribution 